\title{
Challenges to Early Discharge of Patients with Upper Urinary Tract Infections by ESBL Producers: TMP/SMX as a Step-Down Therapy for Shorter Hospitalization and Lower Costs
}

Hye Jin Shi

Jae Hee Wee

Joong Sik Eom (D)

Division of Infectious Diseases, Department of Internal Medicine, Gil Medical Center, Gachon University College of Medicine, Incheon, Korea
Correspondence: Joong Sik Eom Division of Infectious Diseases, Department of Internal Medicine, Gil Medical Center, Gachon University College of Medicine, (2I565) 774 Beon-gil 2I, Namdongdae-ro, Namdong-gu, Incheon, Korea

Tel +82-32-460-2630

Fax +82-32-460-2631

Email helppl@gachon.ac.kr
Background: Urinary tract infections (UTIs) caused by extended spectrum beta-lactamase (ESBL) producing pathogens have increased and are treated with carbapenem in general. Carbapenem use is associated with prolonged hospitalization or daily outpatient visit. The aim of this study was to investigate patients with UTIs by ESBL-producing pathogens for early discharge using an old oral antibiotic, trimethoprim-sulfamethoxazole (TMP-SMX), which is susceptible to ESBL-producing pathogens.

Methods: Data on UTIs caused by ESBL-producing pathogens from a single tertiary hospital were collected retrospectively. Patients who had been treated with intravenous carbapenems or oral TMP/SMX were included. Patients' clinical and microbiological outcomes were compared between oral TMP/SMX and ertapenem treatment groups.

Results: A total of 103 patients were included, 21 of whom had been treated with TMP/ SMX, whereas 82 with ertapenem. Clinical outcomes between the two groups were not significantly different (TMP/SMX: 90.5\%; ertapenem: $84.1 \%, p=0.73$ ). The microbiological cure rate was higher in the TMP/SMX group than in the ertapenem group $(90.5 \%$ vs $58.5 \%$, respectively, $p=0.01)$. The mean duration of hospitalization was significantly shorter in the TMP/SMX group than in the ertapenem group $(8.00 \pm 10.50$ days vs $14.00 \pm 37.00$ days, $p=$ $0.07)$. The mean duration of antibiotic treatment was longer in the ertapenem group than in the TMP/SMX group $(16.45 \pm 4.77$ vs $12.76 \pm 5.37$ days, $p=0.006)$.

Conclusion: For susceptible pathogens, TMP/SMX may enable early discharge as an effective oral antibiotic treatment option for UTIs caused by ESBL-positive pathogens. Additionally, use of oral antibiotics can shorten hospital stays and reduce medical costs.

Keywords: oral antibiotics, ESBL, UTI, TMP/SMX, outpatient treatment

\section{Introduction}

Urinary tract infections (UTIs) are a major health problem that people worldwide suffer from. ${ }^{1,2}$ Estimates from United States registries have shown that UTIs correspond to $0.7 \%$ (standard error $0.1 \%$ ) of ambulatory care globally. ${ }^{3}$ More importantly, UTIs caused by extended spectrum beta-lactamase (ESBL) producing Enterobacteriaceae are increasing, not only in hospital-acquired infections (HAIs) but also in community-onset infections. ${ }^{4-7}$ In the United States, ESBL-producing Enterobacteriaceae accounted for $17.2 \%$ of patients with UTI in $2018-2019 .{ }^{8}$ In Korea, one study in 2014 showed $6.4 \%$ of $E$. coli cases in community acquired UTI 
were ESBL-producing. ${ }^{9}$ Further, it is also estimated that $16.4 \%$ of pediatric UTI cases were caused by ESBL producers. ${ }^{10}$

For ESBL producing strains, there are limited options for antibiotic treatment, and carbapenems have been the drug of choice; however, there are several disadvantages to carbapenems. First, most carbapenems are only available in intravenous formulations. ${ }^{11}$ As a result, patients have to be hospitalized or daily visit the hospital for the parenteral antibiotic treatment even after they have become stable or asymptomatic. Second, extensive use of carbapenems may lead to the selection of some bacteria, such as Stenotrophomonas maltophilia. ${ }^{12}$ Third, carbapenems are broad-spectrum antibiotics; therefore, there is an increased risk of collateral damage, changes in normal microbiomes, and development of resistant strains, such as carbapenemase-producing Enterobacteriaceae (CPE). From an infectious point of view, carbapenem-sparing regimens are required for non-severe multi-drug resistant (MDR) infections, such as UTIs. ${ }^{13}$

Ertapenem is a non-Pseudomonas covering carbapenem used as standard treatment for ESBL-UTIs. It was approved by the food and drug administration (FDA, US) to treat community-acquired pneumonia, complicated UTI, acute pelvic infections, complicated skin and soft tissue infections, and complicated intra-abdominal infection. It is commonly used and maintains higher susceptibility among ESBL-producing Enterobacteriaceae. ${ }^{14}$ Using ertapenem helps reduce hospitalization duration through outpatient antibiotic treatment. However, patients still have to visit the hospital daily to treatment and keep intravenous routes. Moreover, it is difficult to administer parenteral antibiotics during holidays at Korean outpatient clinics.

TMP/SMX, an antibiotic in use since 1974, has been approved to treat UTIs by the United States Food and Drug Administration. Because of the high resistant rate of TMP/SMX to UTI pathogens, it is not recommended as a first line treatment in Korea. However, for that reason, the resistant rate of TMP/SMX to E. coli seems to have become lower. ${ }^{15}$ In real clinical practice, this tendency is much clear, and clinicians are experiencing cases of improvement with oral conversion to TMP/SMX. ${ }^{16} \mathrm{TMP} /$ SMX is available in oral form and can be used in UTIs caused by ESBL-producing bacteria, which are susceptible to it. The aim of this study was to obtain evidence that TMP/SMX therapy (initial or step-down) is comparable to the ertapenem treatment for ESBL-positive TMP/SMXsensitive Enterobacteriaceae. The Institutional Review
Board of the Gil medical center approved the study protocol (GDIRB2020-288). A waver for patient parental consent to review their medical records was granted by the Institutional Review Board. The handling of the patient data confidentiality strictly followed the rules set by the institution and was in compliance with the Declaration of Helsinki.

\section{Methods}

This was a retrospective observational study performed at the Gachon University Gil Medical Center (GMC), a tertiary teaching hospital in Incheon, South Korea from January 2017 to June 2020. This study was approved by the GMC Institutional Review Board. The trial was designed and overseen by infectious diseases specialists. Data were collected and analyzed by infectious disease specialists.

Patients who received TMP/SMX or carbapenem, including ertapenem, for UTI by ESBL producing pathogens from January 2017 to June 2020 were screened for study enrollment. Inclusion criteria were: (i) age $\geq 18$ years, (ii) treatment with TMP/SMX or carbapenem, including ertapenem, for symptomatic UTI by ESBL producing pathogens; and (iii) positive urine cultures with microbiologically confirmed ESBL-producing pathogens resistant to penicillin, monobactams, and cephalosporins. Exclusion criteria were: (i) culture demonstrating nonsusceptibility to TMP/SMX or carbapenem, including ertapenem; (ii) non-UTI bacteremia; and (iii) less than $72 \mathrm{~h}$ of effective antibiotic treatment.

The following data were extracted from the patient's electronic medical record: baseline patient demographics; select comorbid conditions and medical history; previous 12-month history of UTI by ESBL producing pathogens and other healthcare exposures; renal function and serum creatinine at baseline and throughout antibiotic treatment; duration of hospitalization and patient care unit where applicable; duration and history of empirical antibiotic use where applicable; TMP/SMX or ertapenem dose and duration of treatment; initial urine and blood culture results and date; follow-up urine and blood culture results and date where applicable; recurrence, reinfection, and relapse; microbiological outcomes; and clinical outcomes.

Microbiological data collected included date and method of urine culture collection; quantity (in CFU) and identification of urinary specimen(s); culture susceptibilities with minimum inhibitory concentrations (MICs) of select antibiotics (aminoglycosides, fluoroquinolones, 
sulfonamides, and carbapenems), dates of positive blood cultures if applicable, and end of treatment follow-up urine cultures when available. Identification and susceptibility of urinary isolates were determined via conventional methods using the VITEKR2 microbial identification system (bioMerieux, Marcy-l'Etoile, France) and according to the standard of care. Data collected included bacterial genus and species name, MICs, and susceptibility interpretations according to Clinical and Laboratory Standards Institute (CLSI) breakpoints. ${ }^{17}$ ESBL production was verified using the VITEK R2 ESBL test panel.

\section{Definitions}

UTI symptomatology and diagnosis were assessed according to pre-determined criteria using objective information available in the electronic medical records. Characteristics of symptomatic UTIs included increased urinary frequency, dysuria, urgency, abdominal or flank pain, and fever. Upper UTI was defined as acute pyelonephritis or complicated UTI. All definitions coincided with those from the Infectious Diseases Society of America (IDSA) or the World Health Organization (WHO), where applicable. ${ }^{18}$ For patients who could not describe their urinary symptoms, characteristic symptoms may not have been included in the diagnosis. ${ }^{15,19}$

The following definitions were used: 1) febrile: a patient's recorded temperature was $\geq 38^{\circ} \mathrm{C}$. 2) A complicated urinary tract infection was defined as the presence of any of the following conditions: (a) renal abscess; (b) hydronephrosis; (c) presence of any metastatic infections (psoas muscle abscess, brain abscess, infective endocarditis, etc.); (d) presence of urinary stones, urethral strictures, congenital abnormalities; or (e) presence of prostatitis in men. ${ }^{15,19,20}$ 3) Clinical improvement was defined as improvement in symptoms and being afebrile by $72 \mathrm{~h}$ after treatment. 4) Clinical failure was the persistence of symptoms after 7 days of treatment. 5) Intermediate clinical status was neither clinical improvement nor failure. 6) Relapse was defined as developing a UTI with the same pathogen within 30 days, 7) reinfection was developing a UTI with a different organism within 30 days, and 8) recurrence was developing an infection with the same organism within 3 months. 9) Microbiological cure was a negative urine culture at completion of therapy and/or the absence of relapse or reinfection and, lastly, 10) microbiological failure was the development of relapse or reinfection after adequate antimicrobial treatment.

\section{Statistical Analysis}

This study was designed to determine whether TMP/SMX was comparable to ertapenem for the treatment of ESBL UTIs. Results are expressed as mean \pm standard deviation and as incidences in the study population. The Student's $t$-test and Mann-Whitney $U$-test were used to compare continuous variables, and the chi-square test and Fisher's exact test were used for categorical variables. All $\mathrm{P}$ values were two-tailed, and $\mathrm{P}$ values $<0.05$ were considered significant. All statistical analyses were performed using IBM SPSS Statistics for Windows version 24.0 (IBM Corp., Armonk, NY).

\section{Results}

From January 1, 2017, to June 30, 2020, a total of 26,780 UTI cases were retrieved. Among them, 103 were caused by ESBL-producing Enterobacteriaceae and treated with the oral TMP-SMX or intravenous ertapenem. Of all patients, 21 were treated with TMP/SMX, whereas 82 with ertapenem (Figure 1). Treatment groups were divided based on the pathogen-specific treatment, not the empirical treatment. In the ertapenem group, the most common empirical treatment was ertapenem (35cases), followed by cephalosporins (31 cases), quinolones (seven cases), piperacillin/tazobactam (five cases), meropenem (two cases), doripenem (one case), and others (one case). In the TMP/SMX group, the most common empirical treatment was cephalosporins (nine cases), followed by ciprofloxacin (three cases), ertapenem (three cases), TMP/SMX (two cases), meropenem (two cases), imipenem (one case), and piperacillin/tazobactam (one case). The mean durations of empirical antibiotics were 6.29 and 6.88 in ertapenem and TMP/SMX groups, respectively (data not shown). Table 1 shows the baseline characteristics of the two groups. A total of $34 \%$ were male patients, with $42.9 \%$ in the TMP/SMX group and $31.7 \%$ in the ertapenem group. The mean age was 71.1 years and more than $93 \%$ of patients had symptoms when the culture was collected. There were no significant differences between the two groups with respect to age, sex, or initial symptoms. Most of the diagnoses were upper UTIs $(90.5 \%$ in the TMP/SMX group and $90.0 \%$ in the ertapenem group, $P=0.26)$. The mean duration of hospitalization was significantly different between two groups $(8.00 \pm 10.50$ in TMP/SMX group, $14.00 \pm 37.00$ in ertapenem group, $P=0.07)$. The mean duration of antibiotic treatment was also longer in the ertapenem treatment group than in the TMP/SMX group (16.45 \pm 4.77 days vs $12.76 \pm 5.37$ days, 


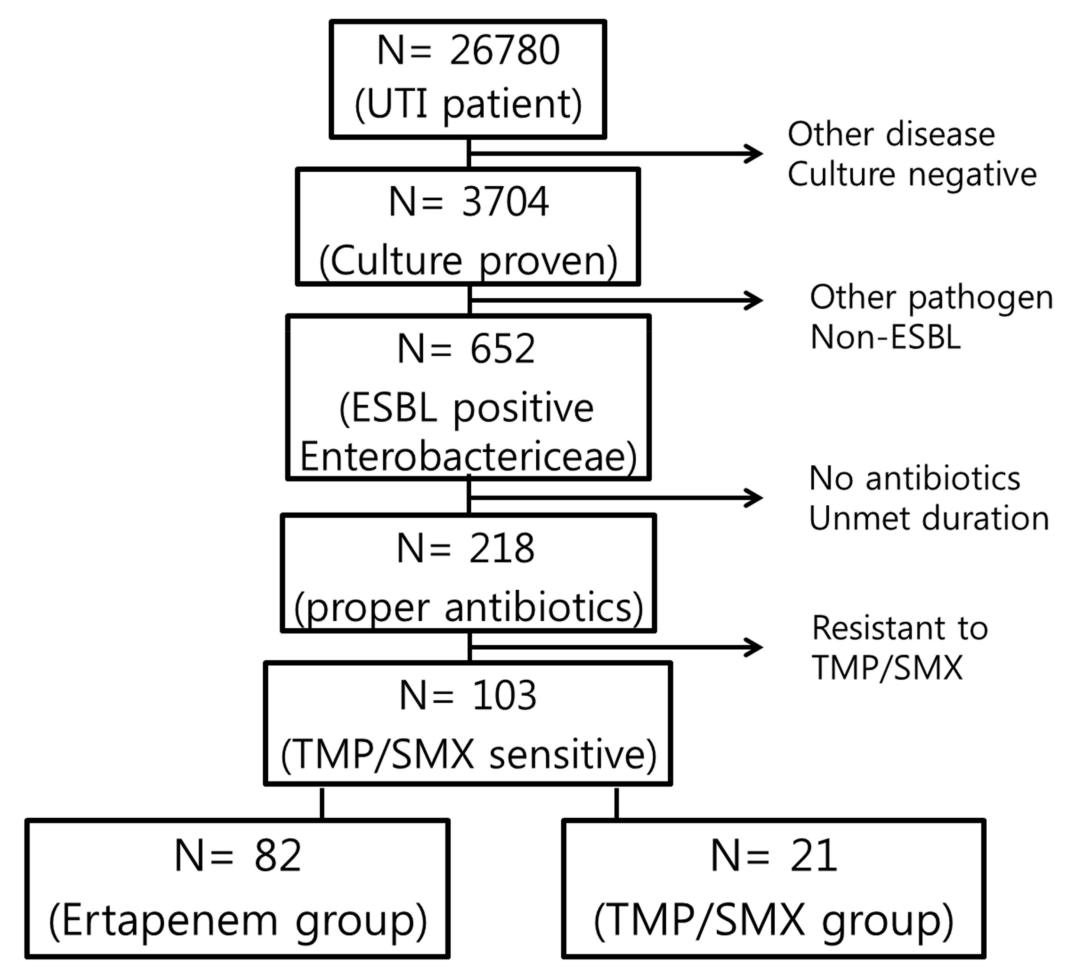

Figure I Flow chart of population in this study.

respectively, $P=0.006$; Table 1 ). We investigated the rate of septic shock from hypotension or ICU admission. There were nine shock cases: two (14.3\%) in the TMP/SMX group and nine $(11.9 \%)$ in the ertapenem group $(p=0.55)$.

Table 2 shows the microbiological characteristics of the two groups. E. coli was the most common pathogen of the initial urine culture in both groups $(85.7 \%$ in the TMP/SMX group and $80.4 \%$ in the ertapenem group), which is consistent with previous studies ${ }^{15,18}$ In the TMP/SMX group, all 21 patients had positive initial urine cultures (Table 2), 18 (85.7\%) of which had E. coli and $2(9.5 \%)$ had P. mirabilis. Initial blood culture in the TMP/SMX group showed $33 \%$ positivity, and E. coli was the most common pathogen. In the ertapenem group, 81 $(98.8 \%)$ of the initial urine cultures were positive, and again, E. coli was the most common pathogen (66 cases, $80.4 \%$ ), followed by $K$. pneumoniae (11 patients, $13.4 \%$ ), P. mirabilis (2 patients, 2.4\%), and E. faecalis (1 patient, $1.2 \%)$. The culture performance rate decreased in both the TMP/SMX and ertapenem groups and in both the urine and blood cultures (Table 2).

We compared the clinical cure rate, clinical improvement after $48 \mathrm{~h}$ of treatment, clinical failure, and death for clinical outcomes (Table 3). Overall, there were no statistically significant differences in clinical outcomes between the TMP/SMX and ertapenem groups (Table 3). Nineteen cases $(90.5 \%)$ in the TMP/SMX group and $69(84.1 \%)$ in the ertapenem group achieved a clinical cure (overall, $85.4 \% ; \mathrm{P}=0.73$ ). The clinical improvement rate after 48 $\mathrm{h}$ of treatment was $23.8 \%$ in the TMP/SMX group and $25.6 \%$ in the ertapenem group $(\mathrm{P}=0.13)$. After $72 \mathrm{~h}$, however, the clinical improvement rate was $90.5 \%$ in the TMP/SMX group and $84.1 \%$ in the ertapenem group ( $\mathrm{P}=$ 0.46) (data not shown). Clinical failure was also similar in both groups $(14.3 \%$ in the TMP/SMX group and $14.6 \%$ in the ertapenem group, $\mathrm{P}=0.99)$. Overall death after 1 month only occurred in the ertapenem group (2 patients, $3.4 \%$ ), but none were associated with the UTI.

The microbiological outcomes of the two groups are shown in Table 4. The microbiological cure rate, negative urine culture at therapy completion, and the absence of relapse or reinfection were significantly higher in the TMP/ SMX group than in the ertapenem group $(90.5 \%$ vs $58.5 \%$, respectively, $\mathrm{P}=0.01)$. The negative follow-up urine culture (FUUC) conversion was similar in both groups (TMP/SMX: 47.6\%; ertapenem: $43.2 \%, \mathrm{P}=0.81)$. Two patients $(9.5 \%)$ in the TMP/SMX group and 12 patients (14.6\%) in the ertapenem group experienced UTIs with the same pathogen within 30 days (relapse), but the difference was not statistically significant $(\mathrm{P}=0.81)$. Recurrence tended to occur somewhat 
Table I Baseline Characteristics of the TMP/SMX and Ertapenem Groups

\begin{tabular}{|c|c|c|c|c|}
\hline & $\begin{array}{l}\text { TMP/SMX } \\
\text { N=2I (\%) }\end{array}$ & $\begin{array}{c}\text { Ertapenem } \\
\mathrm{N}=\mathbf{8 2}(\%)\end{array}$ & $\begin{array}{c}\text { Total } \\
\mathrm{N}=103 \text { (\%) }\end{array}$ & $P$ value \\
\hline Male & $9(42.9)$ & $26(31.7)$ & $35(34)$ & 0.44 \\
\hline Age (year) (median $\pm \mathrm{IQR})$ & $63.67 \pm 21.43$ & $73.16 \pm 58.07$ & $71.11 \pm 52.51$ & 0.46 \\
\hline Symptom at the time of culture & $21(100)$ & $74(94.4)$ & $95(93.1)$ & 0.34 \\
\hline Frequency & $9(42.9)$ & $29(35.4)$ & $38(36.9)$ & 0.61 \\
\hline Urgency & $8(38.1)$ & $15(18.3)$ & $23(22.3)$ & 0.07 \\
\hline Nocturia & $4(19.0)$ & II (I3.4) & $15(14.6)$ & 0.50 \\
\hline Fever & $17(81.0)$ & $63(76.8)$ & $80(77.7)$ & 0.78 \\
\hline Dysuria & $15(71.4)$ & $42(5 \mid .2)$ & $57(55.3)$ & 0.14 \\
\hline CVAT & $9(42.9)$ & $27(32.9)$ & $36(35.0)$ & 0.45 \\
\hline Initial shock & $2(9.5)$ & $9(11.0)$ & II (10.7) & 1.00 \\
\hline Residual urine & $7(33.3)$ & $32(39.0)$ & $39(37.9)$ & 0.80 \\
\hline Rate of upper UTIs & $90.5 \%$ & $90 \%$ & $90.9 \%$ & 0.26 \\
\hline Mean duration of hospitalization (mean \pm SD) & $10.62 \pm 14.32$ & $27.66 \pm 42.02$ & $24.17 \pm 38.42$ & 0.07 \\
\hline $\begin{array}{l}\text { Mean duration of total antibiotics (Intravenous plus Per oral) } \\
\text { (Median } \pm \mathrm{IQR})\end{array}$ & $8 \pm 10.50$ & $14 \pm 37.00$ & $|3.4| \pm 5.46$ & 0.006 \\
\hline Started with selective antibiotics & 0 & $27(45.8)$ & $27(37.0)$ & \\
\hline
\end{tabular}

Abbreviations: TMP/SMX, trimethoprim-sulfamethoxazole; N, number; IQR, interquartile range; CVAT, costovertebral angle tenderness; SD, standard deviation; IQR, interquartile range.

Table 2 Microbiological Characteristics

\begin{tabular}{|c|c|c|c|c|c|c|c|c|}
\hline \multirow[t]{2}{*}{ Pathogen } & \multicolumn{2}{|c|}{ Initial Urine Culture } & \multicolumn{2}{|c|}{ Initial Blood Culture } & \multicolumn{2}{|c|}{ Follow-Up Urine Culture } & \multicolumn{2}{|c|}{ Follow-Up Blood Culture } \\
\hline & $\begin{array}{l}\text { TMP/SMX } \\
\mathrm{N}=21 \text { (\%) }\end{array}$ & $\begin{array}{l}\text { Ertapenem } \\
\mathrm{N}=82(\%)\end{array}$ & $\begin{array}{l}\text { TMP/SMX } \\
\mathrm{N}=21 \text { (\%) }\end{array}$ & $\begin{array}{l}\text { Ertapenem } \\
\mathrm{N}=82 \text { (\%) }\end{array}$ & $\begin{array}{l}\text { TMP/SMX } \\
\text { N=2 I (\%) }\end{array}$ & $\begin{array}{l}\text { Ertapenem } \\
\mathrm{N}=82 \text { (\%) }\end{array}$ & $\begin{array}{l}\text { TMP/SMX } \\
\text { N=2 I (\%) }\end{array}$ & $\begin{array}{l}\text { Ertapenem } \\
\mathrm{N}=82 \text { (\%) }\end{array}$ \\
\hline No growth & 0 & $\mathrm{I}(\mathrm{I} .2)$ & $10(47.6)$ & $36(43.9)$ & $10(47.6)$ & $36(43.9)$ & $4(19.0)$ & $28(34.1)$ \\
\hline E. coli ${ }^{l}$ & I8 (85.7) & $66(80.4)$ & $4(19.0)$ & $14(17.1)$ & $4(19.0)$ & $14(17.1)$ & 0 & 0 \\
\hline K. pneumoniae ${ }^{2}$ & 0 & II (I3.4) & 0 & $2(2.44)$ & 0 & $3(5.1)$ & 0 & 0 \\
\hline P. mirabilis ${ }^{3}$ & $2(9.5)$ & $2(2.4)$ & I (4.7) & $2(2.44)$ & I (4.7) & $2(3.4)$ & 0 & 0 \\
\hline E. faecalis $^{4}$ & 0 & $\mathrm{I}(1.2)$ & I (4.7) & $2(2.44)$ & I (4.7) & $2(3.4)$ & 0 & 0 \\
\hline E. faecium ${ }^{5}$ & 0 & 0 & I (4.7) & $6(7.32)$ & I (4.7) & $6(10.2)$ & 0 & 0 \\
\hline S. maltophilia ${ }^{6}$ & 0 & 0 & 0 & 0 & 0 & $2(3.4)$ & 0 & 0 \\
\hline C. albicans $^{7}$ & 0 & 0 & 0 & 0 & 0 & $3(5.1)$ & 0 & $\mathrm{I}(\mathrm{I} .2)$ \\
\hline Others & 0 & I (I.2) & 0 & $4(4.88)$ & 0 & 0 & 0 & 0 \\
\hline Total & $20(95.3)$ & $82(100)$ & $17(8 \mid)$ & $66(80.5)$ & $17(81)$ & 73 (84.7) & $4(19)$ & $29(35.3)$ \\
\hline Not done & I (4.7) & 0 & $4(19.0)$ & $16(19.5)$ & $4(19.0)$ & $9(15.3)$ & $17(8 \mid .0)$ & $53(64.7)$ \\
\hline
\end{tabular}

Abbreviations: 'E. coli, Escherichia coli; ${ }^{2} \mathrm{~K}$ pneumoniae, Klebsiella pneumoniae; ${ }^{3}$ P. mirabilis, Proteus mirabilis; ${ }^{4} \mathrm{E}$. faecalis, Enterococcus faecalis; ${ }^{5} \mathrm{E}$. faecium, Enterococcus faecium; ${ }^{6} \mathrm{~S}$. maltophilia, Stenotrophomonas maltophilia; ${ }^{7} \mathrm{C}$. albicans, Candida albicans.

Table 3 Clinical Outcomes of the TMP/SMX and Ertapenem Groups

\begin{tabular}{|c|c|c|c|c|}
\hline & TMP/SMX N=2I (\%) & Ertapenem $\mathrm{N}=82(\%)$ & Total $\mathbf{N}=103$ (\%) & $P$ value \\
\hline Clinical cure* & $19(90.5)$ & $69(84.1)$ & $88(85.4)$ & 0.73 \\
\hline Clinical improvement after $48 \mathrm{~h}$ of treatment & $5(23.8)$ & $21(25.6)$ & $26(25.2)$ & 0.13 \\
\hline Clinical failure $^{\#}$ & $3(14.3)$ & $12(14.6)$ & $15(14.6)$ & 0.99 \\
\hline Death within I month & 0 & $2(3.4)$ & $2(2.6)$ & 0.01 \\
\hline
\end{tabular}

Notes: Clinical cure*: clinical improvement after $72 \mathrm{~h}$ of adequate antibiotic treatment. Clinical failure ${ }^{\#}$ : persistence of symptoms after 7 days of adequate antibiotic treatment. Abbreviations: TMP/SMX, trimethoprim-sulfamethoxazole; $\mathrm{N}$, number. 
Table 4 Microbiological Outcomes for the TMP/SMX and Ertapenem Groups

\begin{tabular}{|l|r|r|r|r|}
\hline & TMP/SMX N=2 I (\%) & Ertapenem N=82 (\%) & Total N=103 (\%) & P value \\
\hline Microbiological cure* & $19(90.5)$ & $48(58.5)$ & $67(65.0)$ & 0.01 \\
Negative FUUC & $10(47.6)$ & $35(43.2)$ & $45(44.1)$ & 0.81 \\
Relapse** & $2(9.5)$ & $12(14.6)$ & $14(13.6)$ & 0.73 \\
Reinfection*** & 0 & $14(17.1)$ & $14(13.6)$ & 0.07 \\
Recurrence**** & $2(9.5)$ & $11(13.4)$ & $13(12.6)$ & 0.78 \\
\hline
\end{tabular}

Notes: *Microbiological cure: negative urine culture at the completion of therapy and/or absence of relapse or reinfection. **Relapse: UTI with the same organism within 30 days. *** Reinfection: UTI with a different organism within 30 days. ****Recurrence: infection with same organism within 3 months.

Abbreviations: TMP/SMX, trimethoprim-sulfamethoxazole; N, number; FUUC, follow-up urine culture.

less in the TMP/SMX group than in the ertapenem group, but this difference was also not statistically significant $(9.5 \%$ vs $13.4 \%$, respectively, $\mathrm{P}=0.78$ ). Reinfection was also not statistically significantly different (Table 4).

\section{Discussion}

TMP/SMX is not recommended as an empirical therapy for UTIs because of its high resistance rate in some countries $^{15,18,21}$ While approximately $80 \%$ of UTIs are caused by $E$. coli, ${ }^{22}$ the resistance rate of $E$. coli to $\mathrm{TMP} / \mathrm{SMX}$ is $14.6 \%-37.1 \%$ in developed countries and $54 \%-82 \%$ in developing countries. ${ }^{23}$ However, recent studies revealed that the susceptibility of $E$. coli has changed, showing that TMP/SMX had equal or better susceptibility compared to ciprofloxacin, a first-line treatment option for uncomplicated UTIs. ${ }^{13,23,24}$ Moreover, when drug sensitivity results are present and ESBL-producing pathogens are susceptible to TMP/SMX, there is no reason TMP/SMX cannot be used as a definitive antibiotic for UTIs. ${ }^{25}$

TMP/SMX has an excellent in vitro inhibitory activity against many common respiratory and urinary tract pathogens as well as many nosocomial infection strains. ${ }^{26}$ Our current study reaffirms the usefulness of TMP/SMX in UTI and confirmed that its effects are comparable to those of carbapenems in patients with UTI caused by ESBL-producing Enterobacteriaceae (clinical improvement rate, $>90.5 \%$; microbiological cure rate, 90.5\%). TMP/SMX has some additional advantages over carbapenems. First, it can be administered in both intravenous and oral formulations. As previously mentioned, carbapenems are available only in intravenous formulations. Therefore, patients must be hospitalized and catheterized with an intravenous to receive carbapenem treatment. This is a drawback because hospitalization and fluid maintenance costs accrue. The patients' activities of daily living are restricted even after stabilization of their condition.
Additionally, having an intravenous route increases the risk for phlebitis, thrombosis, and catheter-associated blood stream infections. TMP/SMX can be administered orally; thus, patients can be treated as outpatients.

One study on cost-effectiveness involving patients with bone and joint infections reported that the oral antibiotics group saved $>3600$ dollars on antibiotic, inpatient, and intravenous costs than the intravenous antibiotic groups. ${ }^{27}$ Another study reported markedly reduced expenses when UTI treatment becomes available over the counter. ${ }^{28}$ In our study, the mean hospitalization duration in the TMP/SMX group was significantly shorter than that in the ertapenem group $(8.00 \pm 10.50$ vs $14.00 \pm 37.00, P=0.07)$. The mean treatment duration was also significantly shorter in the TMP/ SMX group than in the ertapenem group $(12.76 \pm 5.37$ vs $16.45 \pm 4.77$ days, $P=0.006)$. Besides, the cost of daily antibiotics was also lower in the TMP/SMX group (in GMC, the daily cost of TMP/SMX was 160 times cheaper than that of ertapenem). Considering the annual UTI incidence, ${ }^{3,29,30}$ the impact of this antibiotic treatment on medical costs is expected to be enormous.

Furthermore, TMP/SMX is a relatively narrowspectrum antibiotic; thus, potential collateral damage is reduced. Carbapenems are known to increase the risk of collateral damage, cause changes in normal microbiomes, and lead to the development of resistant strains such as CPE, which can be difficult to treat. ${ }^{29,31,32}$ While ciprofloxacin has been used as a carbapenem-saving oral treatment option for ESBL-UTIs, it can also cause more collateral damage, such as pseudomembranous colitis, compared to TMP/SMX.

Another possible advantage of TMP/SMX as a carbapenem-saving oral treatment option for ESBLproducing Enterobacteriaceae is that it can be utilized for deep infections. Other oral treatment options, such as fosfomycin, have been highlighted as potential treatments for 
ESBL-UTIs. Fosfomycin is commonly used for ESBL-UTIs and is the recommended first-line treatment for cystitis. ${ }^{15,18}$ However, fosfomycin is only approved for treating lower UTIs due to its pharmacokinetics/pharmacodynamics $(P K / P D)$ properties. In most studies reporting that the effects of fosfomycin are comparable to those of ertapenem, complicated UTIs have been excluded. ${ }^{33}$ One study applied fosfomycin for complicated UTIs and reported an overall microbiological cure rate of $50 \%$ and clinical cure rate of $71 \%,{ }^{34}$ which were much lower than our study findings. The bioavailability of TMP/SMX after oral administration, which has been reported to be $97.5 \%$ $101.8 \%,{ }^{35}$ is excellent. Therefore, TMP/SMX can be used as an oral treatment option for deep infections, such as pneumonia, skin and soft tissue infections, bacteremia, and even bone and joint infections. ${ }^{36,37}$ Our study aimed to target upper UTI cases; thus, $>90 \%$ of the enrolled patients had upper UTIs caused by ESBL-producing pathogens (Table 1). By showing similar levels of clinical and microbial improvement, our results provide evidence supporting the use of TMP/SMX for upper UTIs. Recent literature has highlighted the trend of reinvigorating older antimicrobials to decrease reliance on new agents and to preserve the class efficacy of novel therapies. $^{24}$ TMP/SMX also exhibits an ideal $P K / \mathrm{PD}$ profile and bacterial spectrum for UTI treatments. ${ }^{26}$ Our study results provide evidence supporting TMP/SMX use as outpatient management of ESBL-UTIs. As ESBL-producing bacteria often cause complicated infections with limited treatment options, our data provide significant evidence that TMP/ SMX can be used as an alternative to intravenous carbapenems.

This study has several strengths. First, although most studies comparing the effectiveness between TMP/SMX and carbapenem have included only lower UTI cases, this study mainly included upper UTI cases. Therefore, these results can be applied to both upper and lower UTI treatments. Second, we were able to reliably confirm the effectiveness of TMP/SMX for UTIs by analyzing not only clinical improvement but also microbiological improvement and recurrence in various ways and demonstrating a lack of statistically significant differences between the groups. Third, since many years of real clinical data from a tertiary hospital were collected for this study through a well-designed protocol, a realistic patient group was recruited and comparability was demonstrated.

This study has some limitations. First, due to the retrospective design, there may have been bias during data collection. Given that this study constituted exploratory research without an exact calculation of the sample size in a statistical manner, the study could be insufficiently powered to detect weaker, but potentially clinically significant, effects. Second, a relatively small number of patients (21) were treated with TMP/SMX, and most patients in the current study received initial therapy in the inpatient setting with intravenous antibiotics; therefore, the effects observed also reflect this initial therapy and may have been biased to show similar outcomes. Third, this study did not compare the direct cost-effectiveness between TMP/SMX and ertapenems. However, we tried to compensate for these shortcomings by comparing their hospitalization duration and findings of other studies. Finally, the greatest limitation of our study was that only a few cases susceptible to TMP-SMX could be converted to oral antibiotics. However, susceptibility to TMP/SMX has increased recently, ${ }^{15}$ and among our ESBL producing Enterobacteriaceae cases, the resistant rate of ciprofloxacin was $77 \%$, higher than both ertapenem $(2.7 \%)$ and TMP/SMX (49.5\%). Therefore, we think TMP/SMX will become more useful in near future. It is important to monitor the national unit and our hospital unit for antimicrobial susceptibility data.

TMP/SMX may be used as a step-down oral treatment for UTIs caused by ESBL-producing Enterobacteriaceae, after obtaining microbiological results, confirming sensitivity, and (probably also) ensuring the patient is in a stable clinical condition under appropriate early empiric therapy. From the carbapenem-sparing point of view, TMP/SMX may even be a better choice compared to ertapenem. TMP/SMX can be a cost-effective option, as it leads to lower medical costs in the real-world setting and avoids unnecessary hospitalizations. TMP/SMX can enable early discharge of patients with UTI caused by ESBL-producing Enterobacteriaceae. Large-scale, welldesigned randomized trials are warranted to provide more evidence to support these findings.

\section{Key Message}

- TMP/SMX can be an effective oral antibiotic treatment for UTIs caused by ESBL-positive pathogens.

- Clinical outcomes between the TMP/SMX and carbapenem treatments were equally effective.

- The microbiological cure rate was higher in the TMP/SMX group than in the ertapenem group.

- The duration of hospitalization/antibiotics was significantly shorter in TMP/SMX.

- The use of TMP/SMX can reduce collateral damage and medical costs and shorten hospital stays. 


\section{Funding}

No funding was provided for this study.

\section{Disclosure}

None of the authors have any conflicts of interest to declare.

\section{References}

1. Behzadi P, Urbán E, Matuz M, Benkő R, Gajdács M. The role of gram-negative bacteria in urinary tract infections: current concepts and therapeutic options. Adv Exp Med Biol. 2021;1323:35-69. doi:10.1007/5584_2020_566

2. Gajdács M. The importance of reporting clinical and epidemiological data in urology: local experiences and insights from the international literature. Medicina. 2020;56. doi:10.3390/medicina56110581

3. Schappert SM, Rechtsteiner EA. Ambulatory medical care utilization estimates for 2007. Vital Health Stat 13. 2011;2(169):1-38.

4. Tandogdu Z, Wagenlehner FME. Global epidemiology of urinary tract infections. Curr Opin Infect Dis. 2016;29(1):73-79. doi:10.1097/qco.0000000000000228

5. Zowawi HM, Harris PN, Roberts MJ, et al. The emerging threat of multidrug-resistant Gram-negative bacteria in urology. Nat Rev Urol. 2015;12(10):570-584. doi:10.1038/nrurol.2015.199

6. Benkő R, Gajdács M, Matuz M, et al. Prevalence and antibiotic resistance of ESKAPE pathogens isolated in the emergency department of a tertiary care teaching hospital in hungary: a 5-year retrospective survey. Antibiotics. 2020;9. doi:10.3390/antibiotics9090624

7. Gajdács M, Ábrók M, Lázár A, Burián K. Comparative epidemiology and resistance trends of common urinary pathogens in a tertiary-care hospital: a 10-year surveillance study. Medicina. 2019;55. doi:10.3390/medicina55070356

8. Talan DA, Takhar SS, Krishnadasan A, et al. Emergence of extendedspectrum $\beta$-lactamase urinary tract infections among hospitalized emergency department patients in the United States. Ann Emerg Med. 2021;77(1):32-43. doi:10.1016/j.annemergmed.2020.08.022

9. Seo M-R, Kim S-J, Kim Y, et al. Susceptibility of escherichia coli from community-acquired urinary tract infection to fosfomycin, nitrofurantoin, and temocillin in Korea. J Korean Med Sci. 2014;29 (8):1178-1181. doi:10.3346/jkms.2014.29.8.1178

10. Suh W, Kim BN, Kang HM, Yang EA, Rhim JW, Lee KY. Febrile urinary tract infection in children: changes in epidemiology, etiology, and antibiotic resistance patterns over a decade. Clin Exp Pediatrics. 2020;1:42. doi:10.3345/cep.2020.00773

11. Gajdacs M. Intravenous or oral antibiotic therapy: sophie's choice? Review article. General Int Med Clin Innovations. 2019;5(3):254. doi:10.15761/GIMCI.1000176

12. Gajdács M, Urbán E. Epidemiological Trends and resistance associated with stenotrophomonas maltophilia bacteremia: a 10-year retrospective cohort study in a tertiary-care hospital in hungary. Diseases. 2019;7(2):4788. doi:10.3390/diseases7020041

13. Aslan AT, Akova M. Extended spectrum $\beta$-lactamase producing enterobacteriaceae: carbapenem sparing options. Expert Rev Anti Infect Ther. 2019;17(12):969-981. doi:10.1080/14787210.20 19.1693258

14. Lob SH, Hackel MA, Hoban DJ, Young K, Motyl MR, Sahm DF. Activity of Ertapenem against Enterobacteriaceae in seven global regions-SMART 2012-2016. Eur $j$ Clin Microbiol Infect Dis. 2018;37(8):1481-1489. doi:10.1007/s10096-018-3274-y

15. Kang CI, Kim J, Park DW, et al. Clinical practice guidelines for the antibiotic treatment of community-acquired urinary tract infections. Infect Chemotherapy. 2018;50(1):67-100. doi:10.3947/ ic.2018.50.1.67
16. Gajdács M, Ábrók M, Lázár A, Burián K. Revival of older antibiotics for the therapy of urinary tract infections: old, but gold Part 1: antimicrobial susceptibility of extended-spectrum $\beta$-lactamaseproducing and AmpC $\beta$-lactamase-producing Escherichia coli isolates. Rev Med Microbiol. 2021;32(1):51-56. doi:10.1097/ mrm.0000000000000220

17. Institute CaLS. Performance Standards for Antimicrobial Susceptibility Testing. 30th edition. Guidelines; 2020: 296.

18. Gupta K, Hooton TM, Naber KG, et al. International clinical practice guidelines for the treatment of acute uncomplicated cystitis and pyelonephritis in women: a 2010 update by the Infectious diseases society of america and the european society for microbiology and infectious diseases. Clin Infecti Dis. 2011;52(5):e103-20. doi: $10.1093 / \mathrm{cid} / \mathrm{ciq} 257$

19. Hooton TM, Bradley SF, Cardenas DD, et al. Diagnosis, prevention, and treatment of catheter-associated urinary tract infection in adults: 2009 International clinical practice guidelines from the infectious diseases society of America. Clin Infecti Dis. 2010;50(5):625-663. doi: $10.1086 / 650482$

20. Mendes RE, Castanheira M, Woosley LN, Stone GG, Bradford PA, Flamm RK. Molecular beta-lactamase characterization of Gram-negative pathogens recovered from patients enrolled in the ceftazidime-avibactam Phase 3 trials (RECAPTURE 1 and 2) for complicated urinary tract infections: efficacies analysed against susceptible and resistant subsets. Int J Antimicrob Agents. 2018;52 (2):287-292. doi:10.1016/j.jjantimicag.2018.04.001

21. Bartoletti R, Cai T, Wagenlehner FM, Naber K, Bjerklund Johansen TE. Treatment of urinary tract infections and antibiotic stewardship. Eur Urol Supplements. 2016;15(4):81-87. doi:10.1016/ j.eursup.2016.04.003

22. Auer S, Wojna A, Hell M. Oral treatment options for ambulatory patients with urinary tract infections caused by extended-spectrumbeta-lactamase-producing Escherichia coli. Antimicrob Agents Chemother. 2010;54(9):4006-4008. doi:10.1128/aac.01760-09

23. Kot B. Antibiotic resistance among uropathogenic escherichia coli. Polish j Microbiol. 2019;68(4):403-415. doi:10.33073/pjm-2019-048

24. Dubourg G, Okdah L, Le Page S, Rolain J-M, Raoult D. In vitro activity of 'old antibiotics' against highly resistant Gram-negative bacteria. Int J Antimicrob Agents. 2015;46(6):718-720. doi:10.1016/j. ijantimicag.2015.09.008

25. Gajdács M, Bátori Z, Ábrók M, Lázár A, Burián K. Characterization of resistance in gram-negative urinary isolates using existing and novel indicators of clinical relevance: a 10-year data analysis. Life. 2020;10:10. doi:10.3390/life10020016

26. Cockerill FR, Edson RS. Trimethoprim-sulfamethoxazole. Mayo Clinic Proce. 1991;66(12):1260-1269. doi:10.1016/s0025-6196(12) 62478-1

27. McMeekin N, Geue C, Briggs A, et al. Cost-effectiveness of oral versus intravenous antibiotics (OVIVA) in patients with bone and joint infection: evidence from a non-inferiority trial. Wellcome Open Res. 2019;4:108. doi:10.12688/wellcomeopenres.15314.4

28. Rubin N, Foxman B. The cost-effectiveness of placing urinary tract infection treatment over the counter. J Clin Epidemiol. 1996;49 (11):1315-1321. doi:10.1016/s0895-4356(96)00218-1

29. Zhang Y, Wang Q, Yin Y, et al. Epidemiology of Carbapenem-Resistant Enterobacteriaceae Infections: report from the China CRE Network. Antimicrob Agents Chemother. 2018;62 (2). doi:10.1128/aac.01882-17

30. Critchley IA, Cotroneo N, Pucci MJ, Mendes R. The burden of antimicrobial resistance among urinary tract isolates of Escherichia coli in the United States in 2017. PLoS One. 2019;14(12):e0220265. doi:10.1371/journal.pone.0220265

31. Zimmermann P, Curtis N. The effect of antibiotics on the composition of the intestinal microbiota - a systematic review. $J$ Infect. 2019;79(6):471-489. doi:10.1016/j.jinf.2019.10.008 
32. Gajdács M, Ábrók M, Lázár A, et al. Detection of VIM, NDM and OXA-48 producing carbapenem resistant Enterobacterales among clinical isolates in Southern Hungary. Acta Microbiol Immunol Hung. 2020;67(4):209-215. doi:10.1556/030.2020.01181

33. Veve MP, Wagner JL, Kenney RM, Grunwald JL, Davis SL. Comparison of fosfomycin to ertapenem for outpatient or stepdown therapy of extended-spectrum $\beta$-lactamase urinary tract infections. Int J Antimicrob Agents. 2016;48(1):56-60. doi:10.1016/j. ijantimicag.2016.04.014

34. Bielen L, Likic R. Experience with fosfomycin in the treatment of complicated urinary tract infections caused by extended-spectrum beta-lactamase-producing Enterobacteriaceae. Therapeutic $A d v$ Infect Dis. 2019;6:2049936119858883. doi:10.1177/20499361 19858883
35. Chin TW, Vandenbroucke A, Fong IW. Pharmacokinetics of trimethoprim-sulfamethoxazole in critically ill and non-critically ill AIDS patients. Antimicrob Agents Chemother. 1995;39(1):28-33. doi:10.1128/aac.39.1.28

36. Paul M, Bishara J, Yahav D, et al. Trimethoprim-sulfamethoxazole versus vancomycin for severe infections caused by meticillin resistant Staphylococcus aureus: randomised controlled trial. BMJ. 2015;350: h2219-h2219. doi:10.1136/bmj.h2219

37. Eliakim-Raz N, Hellerman M, Yahav D, et al. Trimethoprim/sulfamethoxazole versus vancomycin in the treatment of healthcare/ventilator-associated MRSA pneumonia: a case-control study. J Antimicrob Chemother. 2017;72(3):882-887. doi:10.1093/jac/ dkw510

\section{Publish your work in this journal}

Infection and Drug Resistance is an international, peer-reviewed openaccess journal that focuses on the optimal treatment of infection (bacterial, fungal and viral) and the development and institution of preventive strategies to minimize the development and spread of resistance. The journal is specifically concerned with the epidemiology of antibiotic resistance and the mechanisms of resistance development and diffusion in both hospitals and the community. The manuscript management system is completely online and includes a very quick and fair peerreview system, which is all easy to use. Visit http://www.dovepress.com/ testimonials.php to read real quotes from published authors. 